\title{
A Kinematic Analysis of Fundamental Movement Skills
}

\author{
Cain C. T. CLARK・Claire M. BARNES・Mark HOLTON \\ Huw D. SUMMERS・ Gareth STRATTON
}

Wundamental movement skills are considered the basic building and sport-specific movement skills required for participation in a variety of physical activities. However, kinematic analyses of fundamental movement has not been performed. The aims of this study were to, (1) characterise the relationship between facets of fundamental movement and, (2) characterise the relationship between overall integrated acceleration and three-dimensional kinematic variables whilst performing fundamental movement skills. Eleven participants $(10 \pm 0.8 \mathrm{y}, 1.41 \pm 0.07 \mathrm{~m}, 33.4 \pm 8.6 \mathrm{~kg}$, body mass index; $16.4 \pm 3.1$ $\mathrm{kgm}^{2}$ ) took part in this study, had anthropometric variables recorded and performed a series of fundamental movement tasks, whilst wearing a tri-axial accelerometer and were recorded using a three-dimensional motion capture system. Maximum shoulder external rotation $\left(^{\circ}\right)$ and maximum shoulder internal rotation velocity $\left({ }^{\circ} \cdot \mathrm{s}^{-1}\right)(\mathrm{r}=0.86, p<0.001)$, mediolateral centre of mass range $(\mathrm{cm})$ and centre of mass coefficient of variation $(\%)(\mathrm{r}=0.83$, $p<0.001)$, maximum stride angle $\left(^{\circ}\right)$ in the jog and walk $(\mathrm{r}=0.74, p=0.01)$ and maximum sprint stride angle and maximum shoulder internal rotation velocity $\left({ }^{\circ} \cdot \mathrm{s}^{-1}\right)(\mathrm{r}=0.67, p<0.02)$ were significantly correlated. Maximum sprint stride angle (hip: $\mathrm{r}=0.96, p<0.001$, ankle: $\mathrm{r}=0.97, p<0.001$ ) and maximum internal rotation velocity (ankle: $\mathrm{r}=0.6, p=0.05$ ) were significantly correlated to overall integrated acceleration. Overall integrated acceleration was comparable between participants (CV: 10.5), whereas three-dimensional variables varied by up to $65 \%$. Although overall integrated acceleration was comparable between participants, three-dimensional variables were much more varied. Indicating that although overall activity may be correspondent, the characteristics of a child's movement may be highly varied.

Keywords: Fundamental movement skills, accelerometry, kinematics, children 


\section{Introduction}

Participation in physical activity is vital for enhancing children's physical, social, cognitive and psychological development (Janssen and Leblanc, 2010). Higher levels of physical activity in children are associated with improved fitness (both cardio-respiratory fitness and muscular strength) (Morrow et al., 2013), enhanced bone health and reduced body fat (Janssen and Leblanc, 2010). Further, children who frequently participate in physical activity demonstrate reduced symptoms of anxiety and depression, and improved self-esteem and confidence (Janssen and Leblanc, 2010).

Accelerometers are the de facto standard in objectively measuring physical activity (Mathie et al., 2004, Van Hees et al., 2012). Commercial devices (such as; ActiGraph, ActiCal) measure activity in the form of 'activity counts', which summarize data over a user-specified epoch, reducing the burden of data management, analysis, and interpretation (Brond and Arvidson, 2015). However, information about the raw accelerometer signal is irretrievably lost and a full picture of physical activity and fundamental movement quality and competency is overlooked (Clark et al., 2016a).

Fundamental movement skills are considered the basic building blocks for movement and provide the foundation for specialized and sport-specific movement skills required for participation in a variety of physical activities. Fundamental movements skills can be categorized as locomotor (e.g., run, hop, jump, leap), object-control (e.g., throw, catch, kick, strike), and stability (e.g., static balance) skills (Gallahue and Ozmun, 2006). There is strong evidence to suggest a positive association between fundamental movement skill competency and physical activity in children (Lubans et al., 2010). Although some studies have relied upon self-report measures of physical activity (Erwin and Castelli, 2008, Graf et al., 2004), a recent review by Barnett et al. (2016) contended the positive relationship between functional movement skills and health related benefits, and highlighted the findings of Holfelder and Schott (2014) and Lubans et al. (2010) who reported predominantly positive associations in their respective systematic reviews. Further, it has been reported, by Cohen et al. (2014), that overall daily physical activity is positively correlated with locomotor and object control competency.

Robust kinematics have been used to successfully analyse multidimensional facets of human movement (Lohman et al., 2011), and in relation to fundamental movement skills, can offer in depth analysis across; object control i.e. throwing velocity and release angle (Wagner et al., 2012), stability i.e. centre of mass movement (Fullam et al., 2013) and locomotion i.e. stride angle (Peveler 
et al., 2012). A kinematic analysis of fundamental movement skills has not been performed prior to this study, but would provide a vital piece of evidence for future research, elucidating the range in fundamental movement skills in a homogenous population, and providing an initial research base to build upon.

Therefore, the aims of this study were to, (1) characterise the relationship between facets of fundamental movement and, (2) characterise the relationship between overall integrated acceleration and three-dimensional kinematic variables whilst performing fundamental movement skills.

\section{Methods}

\section{Participants and settings}

A sample of 11 (four female) participants $(10 \pm 0.8 \mathrm{y}, 1.41 \pm 0.07 \mathrm{~m}, 33.4 \pm 8.6 \mathrm{~kg}$, body mass index; $16.4 \pm 3.1 \mathrm{kgm}^{2}$ ) were recruited to take part in this study. The participants were invited to attend the Swansea University Biomechanics and Motion laboratory on one occasion, had anthropometric variables recorded and performed a series of fundamental movement tasks. This research was conducted in agreement with the guidelines and policies of the institutional ethics committee.

\section{Instruments and procedures}

After familiarisation with the laboratory surroundings, tasks and five minute warm-up, children performed a series of stepwise tasks (Table 1), whilst a three-dimensional motion capture system (Vicon, MX13) recorded all movements. Participants also wore custom built Micro Electro-Mechanical System (MEMS) based devices, which incorporated a tri-axial accelerometer with a +/- 16g dynamic range, 3.9mg point resolution and a 13 bit resolution (with a z-axis amplitude coefficient of variation of 0.004 at $40 \mathrm{hz}$ ) (ADXL345 sensor, Analog Devices). It was housed in a small plastic case and affixed via a Velcro strap to; (1) the lateral malleolar prominence of the fibula of the dominant leg, (2) between the radial and ulnar styloid processes of the dominant hand and (3) mounted to the right side of the hip of each individual and set to record at $40 \mathrm{~Hz}$. 


\begin{tabular}{|c|c|c|}
\hline & Task & Description \\
\hline 1 & Overarm throw & $\begin{array}{l}\text { Using the dominant hand, throwing a standard tennis ball at a } \\
\text { target, five meters away, using an overarm throw. }\end{array}$ \\
\hline 2 & Balance task & $\begin{array}{l}\text { Walking along an up-turned bench, whilst maintaining balance } \\
\text { and control. }\end{array}$ \\
\hline 3 & Walk & Walk at self-selected speed along five metres. \\
\hline 4 & Jog & Jog at self-selected speed along five metres. \\
\hline 5 & Sprint & Run at maximal speed along five metres. \\
\hline
\end{tabular}

\section{Anthropometrics}

Standing and seated stature (measured to the nearest $0.01 \mathrm{~m}$ ) and body mass (to the nearest $0.1 \mathrm{~kg}$ ) were measured using a stadiometer (SECA, Hamburg, Germany), sitting stadiometer (Holtain, Crymych, UK) and digital scales (SECA, Hamburg, Germany), respectively, using standard procedures (Lohmann et al., 1988).

\section{Motion capture}

Motion capture was performed using the Vicon MX13 motion capture system (Vicon Peak, Oxford, UK), including twelve cameras sampling at 200 frames per second. For kinematic analysis, 39 retro-reflective markers of $14 \mathrm{~mm}$ diameter were attached to specific anatomical landmarks (Plug-In Gait Marker Set, Vicon Peak, Oxford, UK) (Figure 1) of every participant. Three-dimensional coordinates of the 39 markers were reconstructed with the Nexus software (Nexus 2.0, Vicon, Oxford, UK) and smoothed using cross validation splines (Woltring, 1986). Both static and dynamic calibrations were performed, and residuals of less than $2 \mathrm{~mm}$ from each camera were deemed acceptable.

The 39 retro-reflective marker were placed at the following anatomical locations; the right forehead (RFHD), left forehead (LFHD), right back of head (RBHD), left back of head (LBHD), the $7^{\text {th }}$ cervical vertebrae $(\mathrm{C} 7)$, the $10^{\text {th }}$ thoracic vertebrae (T10), the clavicle (CLAV), sternum (STRN), the right scapula (RBAK), the left shoulder at the acromio-clavicular joint (LSHO), the right shoulder at the acromio-clavicular joint (RSHO), the left upper arm between shoulder and elbow (LUPA), the right upper arm between shoulder and elbow (RUPA), the lateral epicondyle of the left elbow (LELB), the lateral epicondyle of the right elbow (RELB), the left forearm between the elbow and wrist (LFRA), the right forearm between the elbow and wrist (RFRA), the medial and lateral left wrist (LWRA and LWRB, respectively), the medial and lateral right wrist 
(RWRA and RWRB, respectively), the left hand second metacarpal head (LFIN), the right hand second metacarpal head (RFIN), the left anterior superior iliac spine (LASI), the right anterior superior iliac spine (RASI), the left posterior superior iliac spine (LPSI), the right posterior superior iliac spine (RPSI), the lateral epicondyle of the left knee (LKNE), the lateral epicondyle and the right knee (RKNE), the left thigh between the lateral epicondyle of the knee and greater trochanter (LTHI), the right thigh between the lateral epicondyle of the knee and greater trochanter (RTHI), the left lateral malleolus (LANK), the right lateral malleolus (RANK), the left tibia between the lateral epicondyle of the knee and lateral malleolus (LTIB), the right tibia between the lateral epicondyle of the knee and lateral malleolus (RTIB), the left foot second metatarsal head (LTOE), the right foot second metatarsal head (RTOE), the left heel placed on the calcaneous at the same height as the left foot second metatarsal head (LHEE), the right heel placed on the calcaneous at the same height as the right foot second metatarsal head (RHEE). Which has been used previously with a child population (Leardini et al., 2007, Brostrom et al., 2004).

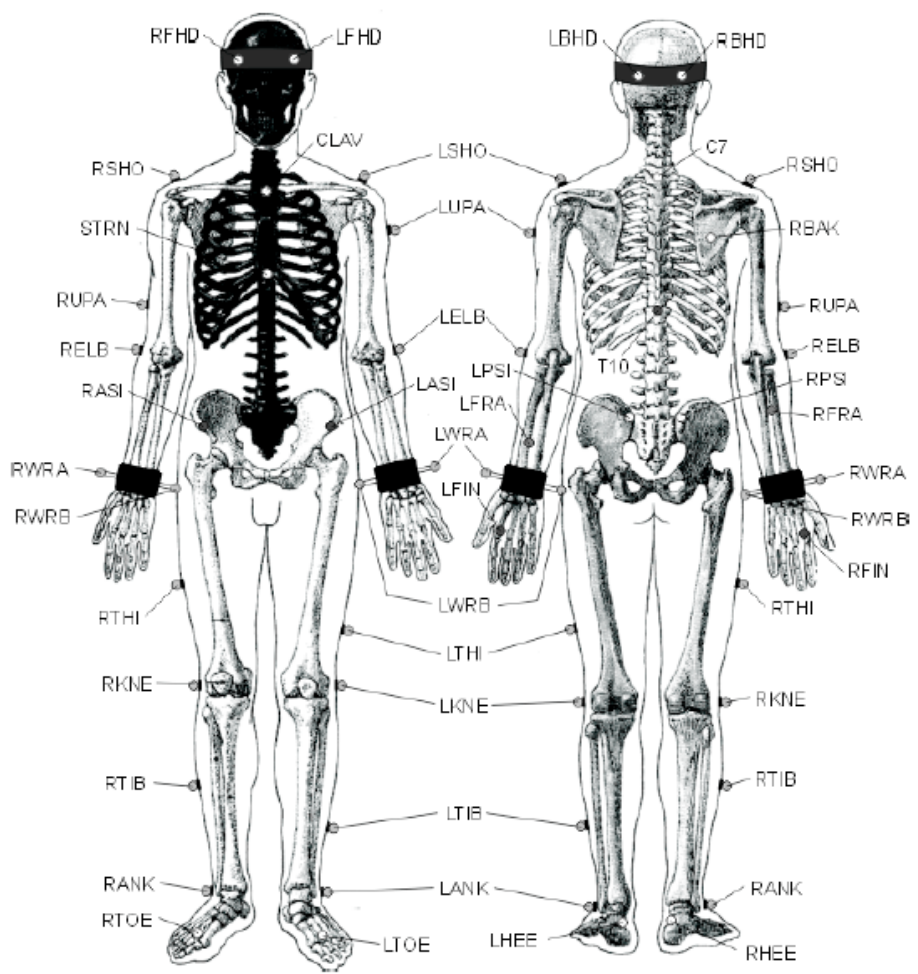

Figure 1. Vicon marker placement 


\section{Data analysis}

\section{MEMS}

Raw acceleration data was uploaded into MatLab (MATLAB version R2016a), where the subsequent movement characteristic; integrated acceleration was derived. The integrated acceleration was determined using an integration of the rectified raw acceleration signal in the radial axis and correspondent to the computation used to derive the standard 'activity counts' by other commercial devices (van Hees et al., 2010).

\section{Vicon}

All corresponding data and video files were first uploaded into Vicon Nexus software and underwent in-depth analysis. Firstly a reconstruct and labelling process was performed, allowing conversion of stereoscopic images into a three-dimensional movement. Once a three-dimensional movement had been established, a functional skeleton calibration was performed and all body segments, joint centres, bone lengths and marker movements were comprehensively modelled and trajectories were manually filtered using Woltring cross validation splines. Every single frame was scrutinised for fluidity and accuracy and marker quality was assessed. Using the three-dimensional reconstruction, maximum elbow flexion $\left(^{\circ}\right)$, maximum shoulder external rotation $\left({ }^{\circ}\right)$, and maximum internal shoulder rotation velocity $\left({ }^{\circ} \cdot \mathrm{s}^{-1}\right)$ were computed for the overarm throw, mediolateral centre of mass range $(\mathrm{cm})$ and coefficient of variation (\%) were computed for the balance task and maximum stride angle (defined as maximum hip extension) was computed for the walk, jog and sprint. Further coefficient of variation between participants for each characteristic was computed. Following this, all kinematic and raw acceleration data was converted into a comma separated values spread sheet for; descriptive statistical analysis, Pearson's product-moment correlation coefficient analysis, and assessed for statistical significance.

\section{Results}

The results of this study found that there were a number of significant relationships within specific movement tasks (throwing, locomotion and balance) and across movement tasks. Descriptive statistics are detailed in table 2. All participants were found to have completed correspondent overall activity for the fundamental movement tasks (Hip: $34.2 \pm 3.5$ counts, Ankle: 50.5 \pm 5.3 counts, CV: 10\%). 


\section{Facets of Fundamental Movement}

For overarm throwing, there was a significant correlation between maximum shoulder external rotation $\left(^{\circ}\right)$ and maximum shoulder internal rotation velocity $\left({ }^{\circ} \mathrm{s}^{-1}\right)(\mathrm{r}=0.86, p<0.001)$. For the balance task there was a significant positive correlation coefficient between mediolateral centre of mass range $(\mathrm{cm})$ and centre of mass coefficient of variation $(\%)(\mathrm{r}=0.83, p<0.001)$. For the locomotion tasks, there was a significant strong positive correlation found between maximum stride angle $\left(^{\circ}\right)$ in the jog and walk $(\mathrm{r}=0.74, p=0.01)$. Finally, there was a significant correlation found between maximum sprint stride angle and maximum shoulder internal rotation velocity $\left({ }^{\circ} \cdot \mathrm{s}^{-1}\right)(\mathrm{r}=0.67, p<0.02)$.

\begin{tabular}{lllllllll}
\hline \multicolumn{3}{c}{ THROW } & & & \multicolumn{2}{l}{ BALANCE } & \multicolumn{2}{l}{ LOCOMOTION } \\
\hline & Max & Max & Max IR & CoM & CoM & Max & Max & Max \\
& ER & EF & velocity & range & CoV & sprint SA & jog SA & walk SA \\
& $\left({ }^{\circ}\right)$ & $\left(^{\circ}\right)$ & $\left(^{\circ} \mathrm{s}^{-1}\right)$ & $(\mathrm{cm})$ & $(\%)$ & $\left({ }^{\circ}\right)$ & $\left({ }^{\circ}\right)$ & $\left(^{\circ}\right)$ \\
Mean & 117.93 & 111.42 & 4021.34 & 42.26 & 0.08 & 27.15 & 19.97 & 14.18 \\
SD & 43.48 & 12.14 & 1667.19 & 12.25 & 0.05 & 3.62 & 3.30 & 2.86 \\
CV $(\%)$ & 37 & 11 & 41 & 21 & 65 & 13 & 17 & 20 \\
\hline
\end{tabular}

Table 2 - Mean $\pm S D$ of fundamental movement variables

*Max ER: maximum shoulder external rotation, Max EF: maximum elbow flexion, Max IR velocity: maximum shoulder internal rotation velocity, CoM range: mediolateral centre of mass range, CoM CoV: centre of mass coefficient of variation, Max sprint $S A$ : maximum sprint stride angle, Max jog SA: maximum jog stride angle, Max walk $S$ A: maximum walk stride angle. CV: coefficient of variation, SD: standard deviation.

\section{Integrated Acceleration vs. Kinematic Variables}

Hip and ankle derived integrated acceleration were positively correlated $(\mathrm{r}=0.97, \mathrm{p}<0.001)$. For locomotion, integrated acceleration at the hip $(\mathrm{r}=0.96$, $\mathrm{p}<0.001)$ and ankle $(\mathrm{r}=0.97, \mathrm{p}<0.001)$ was significantly correlated with maximum sprint stride angle. For overarm throwing, there was a strong positive correlation between maximum internal rotation velocity and integrated acceleration at the ankle $(\mathrm{r}=0.6, \mathrm{p}=0.05)$.

\section{Discussion}

The aims of this study were to, (1) characterise the relationship between facets of fundamental movement and, (2) characterise the relationship between overall integrated acceleration and three-dimensional kinematic variables whilst performing fundamental movement skills. This study identified a number of relationships between and within facets of fundamental movement; maximum shoulder external rotation $\left(^{\circ}\right)$ and maximum shoulder internal rotation 
velocity $\left({ }^{\circ} \mathrm{s}^{-1}\right)(\mathrm{r}=0.86, p<0.001)$, mediolateral centre of mass range $(\mathrm{cm})$ and centre of mass coefficient of variation $(\%)(\mathrm{r}=0.83, p<0.001)$, maximum stride angle $\left({ }^{\circ}\right)$ in the jog and walk $(\mathrm{r}=0.74, p=0.01)$ and maximum sprint stride angle and maximum shoulder internal rotation velocity $\left({ }^{\circ} \mathrm{s}^{-1}\right)(\mathrm{r}=0.67, p<0.02)$ were significantly correlated. This study also identified relationships between maximum sprint stride angle (hip: $\mathrm{r}=0.96, p<0.001$, ankle: $\mathrm{r}=0.97, p<0.001)$ and maximum internal rotation velocity (ankle: $\mathrm{r}=0.6, p=0.05$ ) to overall integrated acceleration.

\section{Facets of Fundamental Movement}

Task specific variables, i.e. overarm throw, balance and locomotion were found to be significantly correlated. The movement required to powerfully throw a ball, overarm, follows a specific developmental sequence (Roberton and Halverson, 1984), where there is a wind-up, stride, arm-cocking, arm acceleration, arm deceleration and follow-through (Dillman et al., 1993). In this sequence of movement, the external and internal rotation of the shoulder is described as one of the most dynamic movements in the human body (Dillman et al., 1993) and is pivotal in power production in overarm throwing. It is therefore necessary for a greater external rotation to produce greater internal rotation velocity.

For stability tasks, it is common to assess this fundamental movement skill using balance beams etc. as a proxy for stability and control (Lubans et al., 2010). In order to competently perform a stability task, it necessitates controlled movement, resulting in minimal mediolateral range, i.e. wobble, and by reducing wobble, centre of mass variation would concomitantly be reduced.

For locomotion tasks, only the jog and walk stride angle were correlated, indicating that an individual's normal gait has minimal bearing on maximum effort gait. The increase in stride angle from volitional walking and jogging was only $\sim 5^{\circ}$, meaning that the increase in speed from walk to jog was only minimal. However the difference in stride angle for the sprint was markedly increased (walk-sprint: $13^{\circ}$ ).

The only significant cross fundamental movement skill relationship was between maximum sprint stride angle and maximum internal rotation velocity. Although these tasks represent very different mechanics and movements, they are both very strongly related to power production. The maximum speed sprint relies upon explosive leg power (Adams et al., 1992) and an overarm throw relies upon power generated, predominantly, from the shoulder and trunk (Roach and Lieberman, 2014). Indicating that if a child is competent and powerful in one fundamental movement skill, it will transfer across skills. It has been shown previously that children who demonstrate competence in locomotion are also 
competent during object-control tasks (Lubans et al., 2010, Cohen et al., 2014). Despite the relationship found, only $45 \%$ of the variance would be explained. However given the nature of developmental sequence involved in overarm throwing, there are a number of trunk, arm and shoulder components that are not present nor required in locomotion, and the step portion of a throw is only a small part of the throwing sequence, meaning a large proportion of movement during internal rotation of the shoulder would be restricted to the upper body (Stodden et al., 2006b, Stodden et al., 2006a).

\section{Integrated acceleration vs. Fundamental Movement}

Locomotion was found to have the greatest correlation to hip and ankle integrated acceleration, this finding can be explained given that the method for calculating integrated activity requires using the radial axis (i.e. along the lower leg towards the origin of motion). Therefore, greater movement along that axis, should result in greater integrated acceleration.

The only other facet of fundamental movement that correlated with integrated acceleration was internal rotation velocity of the overarm throw, however at the ankle only. Given the mechanics of a powerful overarm throw and the developmental sequence of step and trunk action during overarm throwing, there is a strong step action component (Stodden et al., 2006b, Stodden et al., 2006a), where there is a contralateral step forward, and the ipsilateral foot is stretched backwards over half the child's standing stature (Roberton and Halverson, 1984). This large and powerful ipsilateral to contralateral foot range would explain the moderate relationship to integrated acceleration. Nevertheless, only $36 \%$ of the variance was accounted for between these two characteristics.

However, similar to the relationship between maximum sprint angle and internal rotation velocity above, given the action sequence involved for an overarm throw, there are a substantial components that are not present in locomotion, and the ipsilateral step back and contralateral step forward are only minor components of the throw, meaning a large proportion of movement during the throw would be restricted to the upper body, in particular glenohumeral, scapulothoracic, and trunk hyperextension (Stodden et al., 2006b, Stodden et al., 2006a, Dillman et al., 1993).

No other facet of fundamental movement (locomotion, stability, object control) was significantly correlated to integrated acceleration, this is consistent with previously reported literature, where the relationship between object control competency and short activity bouts has been reported to be very weak; $\mathrm{r}=0.11)$ (Cohen et al., 2014). 
Finally, the overall integrated acceleration was comparable between participants (Hip: $34.2 \pm 3.5$ counts, Ankle: 50.5 \pm 5.3 counts), and had a coefficient of variation of $10 \%$, whereas characteristics derived from the threedimensional kinematic analyses varied by up to $65 \%$. Indicating that although overall activity may be the comparable, the characteristics of a child's movement may be noticeably different, even when completing the same activities.

\section{Limitations}

Although the overarm throw was assessed, the exact contribution to total external rotation by each of the shoulder components of glenohumeral, scapulothoracic, and trunk hyperextension was not quantified in this study as it went beyond the scope of the study.

This study utilised a homogenous sample of normal weight, active children, and although their overall integrated acceleration was found to be similar, the facets of fundamental movement were clearly varied, indicating that an overall measure of activity isn't sensitive enough to identify differences in competence or quality of movement.

Fundamental movement skills have previously been linked with health outcomes and physical activity, however, the links have been somewhat tenuous or weak (Lubans et al., 2010, Cohen et al., 2014). It is recommended that more in depth research to dichotomise quality and quantity of activities is needed, which may be achieved through analysing raw acceleration signal features more acutely to reveal information about movement quality and competence across different BMI groups (Clark et al., 2016b).

\section{Conclusion}

The aims of this study were to, (1) characterise the relationship between facets of fundamental movement and, (2) characterise the relationship between overall integrated acceleration and three-dimensional kinematic variables whilst performing fundamental movement skills. This study identified that in a homogenous group of children performing the same fundamental movement tasks, overall integrated acceleration is consistent, whereas quality and competence variables are distinctly varied. This study also demonstrated that characteristics of specific fundamental movements are significantly correlated, as well as between certain movements, which has previously not been done using three-dimensional kinematics. 
Although useful, quantity of activity is an insensitive measure, lacking the ability to identify acute changes, such as; skill acquisition, movement competency, movement quality, motor skill development and developmental disorders. For example, a comprehensive systematic review by Metcalf et al. (2012), involving circa 14,000 participants found physical activity interventions only improve physical activity duration, on average, by 4 minutes per day. The criterion measure of success of an intervention was based on the quantity of accelerometer counts, however the effect on competency or quality of locomotion or other movements, which is of fundamental importance, was overlooked. In this study, the overall activity of participants was comparable, whereas characteristics of their movement were varied (up to 65\%). Indicating that more attention should be given to fundamental differences in movement, as well overall quantity.

Using the raw acceleration signal, activity counts can be computed in an analogous fashion to commercial devices (Van Hees et al., 2012), however there is a clear area for growth in developing beyond simple overall activity quantification, potentially using time-series analysis of raw acceleration to highlight the fundamental differences in similar movements.

Further, as identified by Rudd et al. (2015), although stability skills are recognised as key to fundamental movement, they are scarcely examined, with predominantly locomotion and object control being assessed (Lubans et al., 2010). Therefore it would be recommended that further research analyse stability competence or quality more acutely.

\section{References}

Adams, K., O'shea, J. P., O'shea, K. \& Climstein, M. 1992. The effect of six weeks of squat, plyometric and squat-plyometric training on power production. Journal of Applied Sport Science Research, 6, 36-41.

Barnett, L., Stodden, D. F., Cohen, K. E., Smith, J. J., Lubans, D. R., Lenoir, M., Iivonen, S., Miller, A., Laukkanen, A., Dudley, D. A., Lander, N., Brown, H. \& Morgan, P. J. 2016. Fundamental Movement Skills: An Important Focus. Journal of Teaching in Physical Education, Advance Online Publication.

Brond, J. C. \& Arvidson, D. Sampling frequency affects ActiGraph activity counts. ICAMPAM, 2015 Limerick, Ireland.

Brostrom, E., Hagelberg, S. \& Haglund-Akerlind, Y. 2004. Effect of joint injections in children with juvenile idiopathic arthritis: evaluation by $3 \mathrm{D}$-gait analysis. Acta Paediatr, 93, 906-10. 
Clark, C. C. T., Barnes, C. M., Holton, M. D., Summers, H. D. \& Stratton, G. 2016. Profiling movement quality and gait characteristics according to bodymass index in children (9-11 y). Human Movement Science, 49, 291-300.

Clark, C. C. T., Barnes, C. M., Stratton, G., McNarry, M. A., Mackintosh, K. A. \& Summers, H. D. 2016. A review of emerging analytical techniques for objective physical activity measurement in humans. Sports Medicine. http:// dx.doi.org/10.1007/s40279-016-0585-y.

Cohen, K. E., Morgan, P. J., Plotnikoff, R. C., Callister, R. \& Lubans, D. R. 2014. Fundamental movement skills and physical activity among children living in low-income communities: a cross-sectional study. Int J Behav Nutr Phys Act, 11, 49.

Dillman, C. J., Fleisig, G. S. \& Andrews, J. R. 1993. Biomechanics of Pitching with Emphasis Upon Shoulder Kinematics. Journal of Orthopaedic \& Sports Physical Therapy, 18, 402-408.

Erwin, h. E. \& Castelli, d. M. 2008. National physical education standards: a summary of student performance and its correlates. Res Q Exerc Sport, 79, 495-505.

Fullam, K., Caulfield, B., Coughlan, G. F. \& Delahunt, E. 2013. Kinematic Analysis of Selected Reach Directions of the Star Excursion Balance Test Compared to the Y-Balance Test. J Sport Rehabil.

Gallahue, D. L. \& Ozmun, J. C. 2006. Understanding motor development: infants, children, adolescents, adults. , Boston; United States, McGraw-Hill.

Graf, C., Koch, B., Kretschmann-Kandel, E., Falkowski, G., Christ, H., Coburger, S., Lehmacher, W., Bjarnason-Wehrens, B., Platen, P., Tokarski, W., Predel, H. G. \& Dordel, S. 2004. Correlation between BMI, leisure habits and motor abilities in childhood (CHILT-project). Int J Obes Relat Metab Disord, 28, 22-6.

Holfelder, B. \& Schott, N. 2014. Relationship of fundamental movement skills and physical activity in children and adolescents: A systematic review. Psychology of Sport and Exercise, 15, 382-391.

Janssen, I. \& Leblanc, A. G. 2010. Systematic review of the health benefits of physical activity and fitness in school-aged children and youth. Int J Behav Nutr Phys Act, 7, 40. 
Leardini, A., Sawacha, Z., Paolini, G., Ingrosso, S., Nativo, R. \& Benedetti, M. G. 2007. A new anatomically based protocol for gait analysis in children. Gait Posture, 26, 560-71.

Lohman, E. B., 3Rd, Balan Sackiriyas, K. S. \& Swen, R. W. 2011. A comparison of the spatiotemporal parameters, kinematics, and biomechanics between shod, unshod, and minimally supported running as compared to walking. Phys Ther Sport, 12, 151-63.

Lohmann, T. G., Roche, A. F. \& Martorell, R. 1988. Anthropometric Standardization Reference Manual, Champaign, IL, Human Kinetics.

Lubans, D. R., Morgan, P. J., Cliff, D. P., Barnett, L. M. \& Okely, A. D. 2010. Fundamental movement skills in children and adolescents: review of associated health benefits. Sports Med, 40, 1019-35.

Mathie, M. J., Coster, A. C., Lovell, N. H. \& Celler, B. G. 2004. Accelerometry: providing an integrated, practical method for long-term, ambulatory monitoring of human movement. Physiol Meas, 25, R1-20.

Metcalf, B., Henley, W. \& Wilkin, T. 2012. Effectiveness of intervention on physical activity of children: systematic review and meta-analysis of controlled trials with objectively measured outcomes (EarlyBird 54). BMJ, 345, e5888.

Morrow, J. R., Jr., Tucker, J. S., Jackson, A. W., Martin, S. B., Greenleaf, C. A. \& Petrie, T. A. 2013. Meeting physical activity guidelines and health-related fitness in youth. Am J Prev Med, 44, 439-44.

Peveler, W. W., Shew, B., Johnson, S. \& Palmer, T. G. 2012. A kinematic comparison of alterations to knee and ankle angles from resting measures to active pedaling during a graded exercise protocol. J Strength Cond Res, 26, 3004-9.

Roach, N. T. \& Lieberman, D. E. 2014. Upper body contributions to power generation during rapid, overhand throwing in humans. J Exp Biol, 217, 2139-49.

Roberton, M. A. \& Halverson, L. E. 1984. Developing children - Their changing movement: A guide for teachers., Philadelphia, Lea \& Febiger.

Rudd, J. R., Barnett, L. M., Butson, M. L., Farrow, D., Berry, J. \& Polman, R. C. 2015. Fundamental Movement Skills Are More than Run, Throw and Catch: The Role of Stability Skills. PLoS One, 10, e0140224. 
Stodden, D. F., Langendorfer, S. J., Fleisig, G. S. \& Andrews, J. R. 2006A. Kinematic constraints associated with the acquisition of overarm throwing part I: step and trunk actions. Res Q Exerc Sport, 77, 417-27.

Stodden, D. F., Langendorfer, S. J., Fleisig, G. S. \& Andrews, J. R. 2006B. Kinematic constraints associated with the acquisition of overarm throwing part II: upper extremity actions. Res Q Exerc Sport, 77, 428-36.

Van Hees, V., Gorzelniak, L., Leon, E., Eder, M., Pias, M., Taherian, S., Ekelund, U., Renstrom, F., Franks, P., Horsch, A. \& Brage, S. A method to compare new and traditional accelerometry data in physical activity monitoring. World of Wireless Mobile and Multimedia Networks (WoWMoM), 2012 Montreal, QC, Canada. 1-6.

Van Hees, V., Pias, M., Taherian, S., Ekelund, U. \& Brage, S. A method to compare new and traditional accelerometry data in physical activity monitoring. IEEE International Symposium A World Wireless, Mobile Multimedia Network, 2010.

Wagner, H., Pfusterschmied, J., Tilp, M., Landlinger, J., Von Duvillard, S. P. \& Muller, E. 2012. Upper-body kinematics in team-handball throw, tennis serve, and volleyball spike. Scand J Med Sci Sports.

Woltring, H. J. 1986. A Fortran Package for Generalized, Cross-Validatory Spline Smoothing and Differentiation. Advances in Engineering Software and Workstations, 8, 104-113.

Cain C. T. CLARK, MSc is a doctoral candidate in field of Sport Science at Swansea University, UK. Clark's research focuses on the application of sensors in physical activity, movement skills and gait quality in a paediatric population.

\section{Corresponding author}

Cain C. T. Clark, MSc

College of Engineering,

Bay Campus,

Swansea University,

Fabian Way,

Swansea, Wales,

SA1 8EN

Email: 801344@swansea.ac.uk 
Claire M. BARNES, MSc is a doctoral candidate in the college of Engineering at Swansea University, UK. Barnes' research focuses on applying novel techniques to analyse human movement.

Mark HOLTON, PhD is am engineering research officer at Swansea University, UK. Holton specialises in electronics and programming and his latest work has involved the further development of animal data loggers for further use in Sports Science for monitoring injury and recovery mechanisms, and working across Physics and Astronomy.

Huw D. SUMMERS, PhD is a professor of nanotechnology for health and head of the Multidisciplinary Nanotechnology Centre (MDC) at Swansea University, UK. Professor Summers is internationally recognised for his research excellence, which focuses on two areas: metrologies for cell analysis (cytometry) and the development of nanoparticle-based diagnostics and therapeutics (nanomedicine).

Gareth STRATTON, PhD is a professor of paediatric exercise science and head of the research centre in Applied Sports, Technology, Exercise and Medicine (A-STEM) at Swansea University, UK. Professor Stratton's research interests and expertise are in; paediatric Exercise Science, physical growth and development in health and sports performance, sensors in physical activity measurement, physical activity and fitness and health interventions. 